\title{
A Laser Frequency Comb System for Absolute Calibration of the VTT Echelle Spectrograph
}

\author{
H.-P. Doerr ${ }^{1} \bullet$ T. Steinmetz ${ }^{2,3} \bullet$ R. Holzwarth ${ }^{2,3}$ \\ T. Kentischer ${ }^{1} \bullet$ W. Schmidt ${ }^{1}$ \\ ${ }^{1}$ Kiepenheuer-Institut für Sonnenphysik, Freiburg, Germany \\ 2 Max-Planck-Institut für Quantenoptik, Garching, Germany \\ 3 Menlo Systems GmbH, Martinsried, Germany
}

\author{
Submitted to SolarPhysics: 30 November 2011, Accepted: 24 February 2012. Published Online: \\ 28 March 2012 \\ This reprint was typeset by the Author with permission by Springer/SolarPhysics. \\ The final publication is available at: \\ http://www.springerlink.com/content/3j087q0121n770h1 \\ DOI 10.1007/s11207-012-9960-5
}

\begin{abstract}
A wavelength calibration system based on a laser frequency comb (LFC) was developed in a co-operation between the Kiepenheuer-Institut für Sonnenphysik, Freiburg, Germany and the Max-Planck-Institut für Quantenoptik, Garching, Germany for permanent installation at the German Vacuum Tower Telescope (VTT) on Tenerife, Canary Islands. The system was installed successfully in October 2011. By simultaneously recording the spectra from the Sun and the LFC, for each exposure a calibration curve can be derived from the known frequencies of the comb modes that is suitable for absolute calibration at the meters per second level. We briefly summarize some topics in solar physics that benefit from absolute spectroscopy and point out the advantages of LFC compared to traditional calibration techniques. We also sketch the basic setup of the VTT calibration system and its integration with the existing echelle spectrograph.
\end{abstract}

\section{Introduction}

\subsection{The Need for High-Accuracy Absolute Calibration in Solar Spectroscopy}

The wavelength of spectral lines formed on the solar photosphere is influenced by many well-known processes. Some of the observed wavelength shifts are due to a true Doppler shift introduced by motion of the plasma on the solar photospheric surface, e.g., rotation $\left(2000 \mathrm{~m} \mathrm{~s}^{-1}\right)$, granulation $\left(500 \mathrm{~m} \mathrm{~s}^{-1}\right)$, and solar oscillations $\left(500 \mathrm{~m} \mathrm{~s}^{-1}\right)$. The convective blueshift and gravitational redshift introduce wavelength shifts on the order of $500 \mathrm{~m} \mathrm{~s}^{-1}$, while magnetic fields alter the magnitude of the convective blueshift itself, but these 
effects may not be interpreted as Doppler shifts. Also, the rapidly changing line-of-sight velocity between an observer on the Earth and the observed feature on the Sun amounts to roughly $1000 \mathrm{~m} \mathrm{~s}^{-1}$.

To measure small-scale velocities of the plasma on the solar surface, all these effects must be separated carefully. Sensitive and stable instruments are needed to measure Doppler velocities of a few meters per second on top of the solar "noise" because long averaging times are required.

While most processes can be characterized with spectroscopic measurements that are stable to an arbitrarily chosen zero point, precise absolute calibration is crucial to some measurements of small-scale Doppler velocities. We shall now summarize some of the topics in solar physics that would benefit from high-precision absolute wavelength measurements.

\subsubsection{Convective Blueshift and Limb Effect}

The convective blueshift is a shift of spectral lines of up to $500 \mathrm{~m} \mathrm{~s}^{-1}$ caused by solar granulation (Beckers and Nelson, 1978). The blueshift is strongest at the disk center and shows a center-to-limb variation, which is also known as the "limb effect" (see, e.g. Balthasar, 1984). The limb effect further complicates Doppler measurements on the solar surface, as it cannot be averaged out by taking longer time series. Instead, a precise characterization of the limb effect depending on the position on the solar disk and the spectral line is required to correct measurements. However, so far, absolute measurements of the limb function have been limited to a few lines where accurate wavelength references are available (e.g. Kentischer and Schroeter, 1991) and model computations are employed for calibration purposes (de la Cruz Rodríguez, Kiselman, and Carlsson, 2011). For any precise spectroscopic measurement of the meridional flow as described below, a preceding determination of the limb function is a prerequisite, as the effect could also vary with the solar activity.

\subsubsection{Meridional Motion}

The meridional motion is a weak, poleward directed flow on the solar surface with an am- plitude of about $20 \mathrm{~m} \mathrm{~s}^{-1}$. At high latitudes, the flow is expected to sink to deeper layers and turn equatorward, resulting in a circulation whose revolution time scale might be connected to the 11-year solar magnetic cycle (see, e.g. Dikpati and Gilman, 2007). So far, most mea- surements of the meridional flow are derived from feature tracking or helioseismic methods. However, the velocity derived from feature tracking is different from the true plasma mo- tions, and helioseismology does not give reliable results at the surface due to noise from solar granulation. Thus, Doppler velocities derived from absolute calibrated high-resolution spectra would complement the existing measurements. 


\subsubsection{Sun-as-a-Star Radial Velocity vs. Solar Activity}

Stellar radial velocity measurements have now reached a precision well below the meters per second scale (eg. Lovis et al., 2008), and the required $5 \mathrm{~cm} \mathrm{~s}^{-1}$ sensitivity to detect Earth-like planets orbiting Sun-like stars seems to be feasible. In this radial velocity regime, Doppler noise from stellar activity usually dominates the detection limit, as it readily exceeds the Doppler signal from small orbiting planets and the instrument stability. Studying how the solar activity affects the radial velocity signal of the Sun thus is of great importance in detecting signatures of Earth-like exoplanets orbiting Sun-like stars. Detailed studies were readily carried out on this subject (e.g. Lagrange, Desort, and Meunier, 2010; Makarov, 2010), and high-resolution solar spectra with an absolute wavelength calibration will be useful to verify model calculations and measurements taken with different instruments.

\subsection{Wavelength Calibration Techniques}

Several traditional calibration techniques commonly used in astronomy provide reference lines that are stable at the meters per second level. However, for absolute wavelength calibration, stability of the used reference lines is necessary but not sufficient, as their absolute laboratory wavelength must also be known with a corresponding accuracy.

The ideal wavelength reference for spectrograph calibration provides a dense spectrum of equidistant emission lines of equal intensity with a line width smaller than the instrument's resolution. The frequencies of the reference lines should be derivable from fundamental physics so that the calibration source becomes exchangeable without affecting the calibration.

Different calibration techniques were compared with frequency comb calibrators by Murphy et al. (2007) for their suitability for extremely stable night-time spectrograph calibration. They concluded that so far only frequency combs allow one to exploit the limits of existing and future high-precision spectrographs. While most of their findings are also applicable for solar spectroscopy, some aspects are different in this field.

In night-time spectroscopy, thorium-argon lamps are commonly used for calibration. The Th-Ar spectrum covers the visible spectrum with thousands of sharp lines, many of which are known with an accuracy of about $10 \mathrm{~m} \mathrm{~s}^{-1}$ (Lovis and Pepe, 2007). But while the dim intensity of these lamps is sufficient for typical integration times of several minutes in night-time astronomy, solar spectroscopy usually deals with exposure times well below one second even at very high spectral resolution. The use of iodine cells is limited to the spectral range between 500 and $630 \mathrm{~nm}$. The dense $\mathrm{I}_{2}$ spectrum blends with solar lines, and deconvolution techniques must be employed to recover the true solar spectrum from the measured data (Koch and Woehl, 1984). While both Th-Ar and $\mathrm{I}_{2}$ calibrators provide very stable references with a reasonable number of calibration lines, a major drawback is the huge variation in intensity of their emission/absorption lines. Also, their atomic parameters are not known precisely enough for absolute calibration at 
a few meters per second.

Recently, several frequency comb-based calibration systems were proposed for astronomical spectrographs (e.g. Murphy et al., 2007; Braje et al., 2008; Li et al., 2008), and a few test setups readily demonstrated their superior performance for wavelength calibration (Steinmetz et al., 2008; Benedick et al., 2010; Wilken et al., 2010). Wilken et al. (2010) also showed that with grating spectrographs it is not sufficient to rely on a few calibration lines only, as aberrations of the spectrograph optics itself or even inter-pixel irregularities of the CCD could introduce deviations from the best-fit pixel-to-frequency curve that exceed several $10 \mathrm{~m} \mathrm{~s}^{-1}$.

\section{The VTT Wavelength Calibration System}

The feasibility of calibrating astronomical spectrographs with frequency combs was demonstrated for the first time by Steinmetz et al. (2008) using the Vacuum Tower Telescope (VTT) echelle spectrograph with a frequency comb operated in the infrared range. During the last two years, a laser frequency comb-based wavelength calibration system was developed for the VTT spectrograph in a cooperation between the Kiepenheuer-Institut für Sonnenphysik, Freiburg, Germany and the Max-Planck-Institut für Quantenoptik, Garching, Germany. The system was planned to cover a spectral range of at least $530 \pm 50 \mathrm{~nm}$ in the visible with a mode separation of about $5 \mathrm{pm}$. The VTT spectrograph has a spectral resolution of $\frac{\lambda}{\delta \lambda}>10^{6}(0.5 \mathrm{pm} @ 500 \mathrm{~nm})$. For our application the rather large mode separation is required, because it easily allows us to unambiguously identify the frequencies of the comb lines by comparison with a nearby line in the solar spectrum.

For the details of frequency comb generation and their application in astronomy we refer to Holzwarth et al. (2000); Murphy et al. (2007); Steinmetz et al. (2008) and Wilken (2010). Here we merely describe the basic principle of our astro-comb setup and its integration with the spectrograph.

\subsection{Optical Frequency Combs}

The spectrum of a mode-locked femtosecond laser forms a comb-like structure of equidistantly spaced modes with a frequency separation that is equal to the repetition rate $f_{\text {rep }}=$ $1 / T$ of the laser, where $T$ is the round-trip time of the pulse in the laser resonator. Inequality of group and phase velocity in the resonator introduces a nonzero offset frequency $\left|f_{0}\right|<f_{\text {rep }}$ of the laser modes so that the frequency of the $n$th mode,

$$
f_{n}=f_{0}+n f_{\text {rep }}
$$

is completely defined by the two radio frequencies (RFs) $f_{0}$ and $f_{\text {rep }}$. Equation (1) establishes a direct link between the RFs and optical spectrum. Both frequencies can be measured and controlled very precisely, enabling the generation of optical frequencies with the same precision that previously was only available to RF electronics. While $f_{\text {rep }}$ 


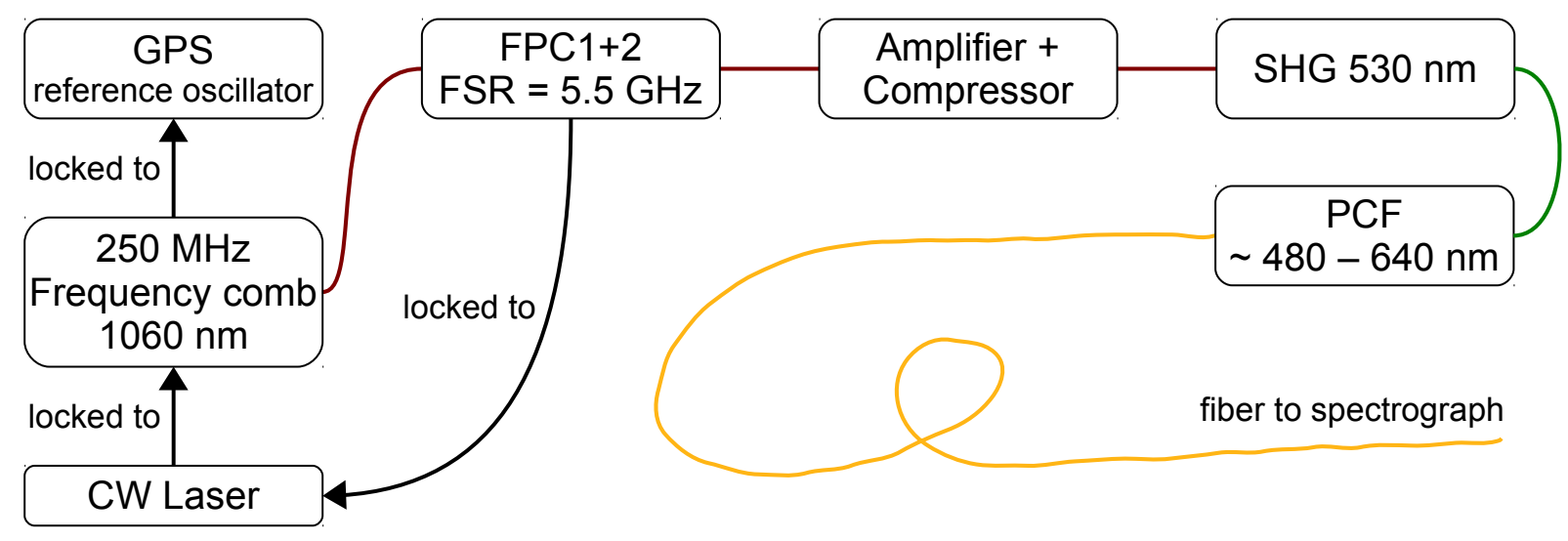

Figure 1: Schematic view of the VTT astro-comb.

can be measured straightforwardly with a fast photo-diode, determination of $f_{0}$ requires additional effort. One possibility is to use an $f: 2 f$-interferometer where the red part of the LFC spectrum is frequency doubled and the beat note with the blue part then yields $f_{0}$. This requires the comb spectrum to span at least one octave and was first accomplished by spectral broadening of the comb in a photonic crystal fiber (PCF) (Holzwarth et al. 2000). The equidistant spacing of the comb modes can be derived from theory, and no deviation from the equidistancy could be detected experimentally at a level of a few parts in $10^{16}$.

While frequency combs are now routinely used in many fields in metrology and spectroscopy, their application in astronomy was delayed until recently for two reasons: 1) the fundamental repetition rate of the comb lasers (typically of the order of $100 \mathrm{MHz}$ or approximately $0.1 \mathrm{pm} @ 500 \mathrm{~nm}$ ) is much to small to be resolved even by high-resolution astronomical spectrographs and, 2), the wavelength coverage of the comb spectrum is too narrow to be useful for multipurpose astronomical spectroscopy.

\subsection{The VTT Astro-Comb}

There are several solutions to the problems mentioned above. The approach we follow is to filter the the fundamental repetition rate of the frequency comb with Fabry-Pérot cavities (FPCs) to a higher effective repetition rate $f_{\text {rep,astrocomb }}=m f_{\text {rep }}$ where $m$ is the integer ratio of the free spectral range (FSR) of the cavity and $f_{\text {rep }}$. The filtered spectrum is amplified, converted to the visible spectrum, and finally broadened in a PCF. A schematic view of the setup is shown in Figure 1.

The heart of the VTT astro-comb is a commercial frequency comb system (Menlo Systems FC1000). It consists of a ytterbium fiber laser operated at $1060 \mathrm{~nm}$ with a repetition rate of $250 \mathrm{MHz}$. The repetition rate and offset frequency of the laser are phase-locked to a GPS disciplined reference oscillator with a stability and accuracy of $10^{-12} @ 1 \mathrm{~s}$. While with Ti:Sa lasers, a higher fundamental repetition rate of $\approx 1 \mathrm{GHz}$ would be possible, 
fiber lasers have the big advantage of turnkey operation, allowing for unattended operation by non-experts.

The comb is filtered with $m=22$ with two identical planoconcave FPCs with a free spectral range of $5.5 \mathrm{GHz}$ and a finesse of approximately 500. By using two cavities we obtain a suppression of the nearest $250 \mathrm{MHz}$ sidemodes by more than $60 \mathrm{~dB}$ (Steinmetz et al., 2009). The plane mirrors of the cavities are piezo-driven for very fast adjustment of the resonator length. Both cavities are locked with the Pound-Drever-Hall method (e.g. Black, 2001) to the transmission signal of a continuous wave (CW) laser with a polarization state that is orthogonal to that of the comb laser. The CW laser itself is locked to one of the comb modes transmitted by the cavities. Since the CW laser is comparably stable, the cavities stay locked even when the seed laser is switched off or blocked for a short time. As the cavities might drift out of the piezo range due to changes in pressure or temperature, a coarse tracking system with thermoelectrial heaters is implemented. To compensate for losses of the laser power in the filter cavities, the signal from the seed laser is amplified with core-pumped fiber amplifiers before passing the cavities. After filtering, a high-power double-clad fiber amplifier and a pulse compressor are inserted in the beam as the second-harmonic generation ( $\mathrm{SHG}$ ) requires high pulse powers to be efficient and enough power must also be available afterwards for spectral broadening in the PCF. We achieve good spectral broadening with an average power of about $1.5 \mathrm{~W}$ before the SHG stage.

Up to the pulse compressor, all optical components are fiber coupled except for a freespace section in the FPCs. This makes the optical alignment of the system very robust; readjustment will probably only be necessary during the regular telescope maintenance periods.

To enhance the non-linear effects that enable the spectral broadening, the PCF is tapered (Stark et al., 2011). The characteristics of the broadening strongly depend on the fiber which can not yet be manufactured with completely identical properties. With most of the PCFs we have tried so far, broad spectra ranging from approximately 480 to $640 \mathrm{~nm}$ could be obtained with enough power for short exposure times that are still limited by the flux from the sunlight.

\subsection{Integration with the Spectrograph}

In solar physics spectroscopy usually also involves imaging of features on the solar surface. By scanning the spectrograph slit, images can be obtained with high spatial and spectral resolution (at the cost of temporal resolution). However, this further complicates the use of external wavelength calibration standards, as the calibration light must be combined with the sunlight so that identical illumination of the spectrograph grating from both light sources is ensured. This is probably the most critical issue of the complete setup. Night-time astronomy is different in this aspect. Stars are always unresolved point sources; the star light and calibration light can be fed to the spectrograph in two optical fibers which provide excellent stability and any offset between both fibers can easily be 
measured.

For observations that rely on the spatial information provided by the slit spectrograph we are currently developing a slit illumination unit that will allow us to align the calibration light fed to the spectrograph in a singlemode fiber with respect to the optical axis of the telescope. Telescope and fiber pupil are imaged on a CCD camera, and the position of both can be tracked and aligned with a precision that translates to a few meters/second. While this would still limit our ability for absolute calibration, the stability of the calibration itself is not affected.

To characterize the limits of the spectrograph calibration we are also preparing an experimental all-fiber setup where integrated sunlight from a full-disk telescope and the calibration light is fed to the spectrograph in the same single mode fiber. By using a single mode fiber, a perfect mode match between calibration light and sunlight can be guaranteed, eliminating any systematic effects related to unequal illumination of the grating with sunlight and calibration light. This setup then can also be used for high-precision Sun-as-a-Star spectroscopy.

\subsection{Expected Performance}

The photon-noise limited uncertainty in velocity information that can be extracted from a single line can be estimated (Brault, 1987) by the relation

$$
\sigma_{v}=A \frac{\mathrm{FWHM}}{\operatorname{SNR} \sqrt{n}}
$$

where $A$ is a weighting factor that depends on the line shape $(0.41$ for a gaussian according to Murphy et al. (2007)), FWHM is the full width at half maximum of the line in meters/second, SNR is the peak signal-to-noise-ratio of the line profile, and $n$ is the number of pixels per FWHM at which the spectrum is sampled on the CCD. For the VTT spectrograph $\sigma_{v}$ corresponds to 0.7 meters/second at a moderate SNR of 100 .

As the VTT spectrograph is neither temperature nor pressure stabilized, drifts of the order of $0.1 \mathrm{pm}(50 \mathrm{~m} / \mathrm{s})$ per hour are common. While this drift seems rather large, at exposure times of one second it is still below the detection limit and can easily be tracked when the calibration spectrum is recorded simultaneously with the science data. Figure 2 shows a test exposure taken during the installation campaign in October 2011. In the upper panel, a part of the solar spectrum at about $530 \mathrm{~nm}$ is shown with the calibration spectrum from the LFC at the lower edge of the CCD image. At $530 \mathrm{~nm}$, the PCF introduces strong intensity variations that however quickly flatten with departure from the central wavelength of the frequency-doubled laser comb.

\section{Conclusion and Outlook}

A frequency comb-based calibration system was developed for the VTT spectrograph and successfully installed at the telescope in October 2011. The astro-comb allows for 

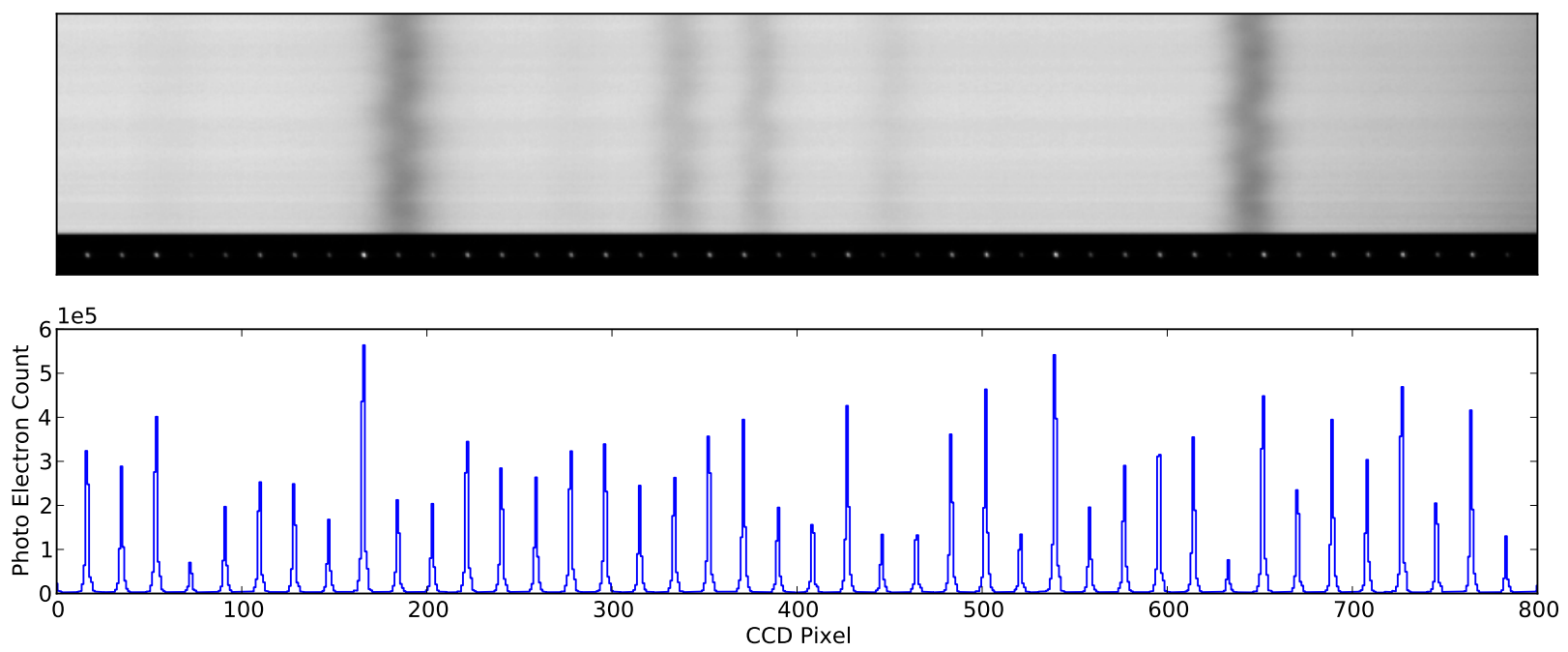

Figure 2: A part of the solar spectrum at about $530 \mathrm{~nm}$ simultaneously recorded with the calibration spectrum from the frequency comb on 8 Oct 2011 17:35 UTC near the disk center (upper panel). The lower panel shows a cut through the LFC spectrum in horizontal direction at the same image scale.

absolute calibration of solar spectroscopic measurements in a broad wavelength range between approximately 480 and $640 \mathrm{~nm}$. As many spectroscopic measurements in solar physics suffer from unreliable calibration, we see great potential for a very highresolution spectrograph in combination with an absolute wavelength calibration system. The system is currently being tested and characterized thoroughly; the first scientific campaigns are scheduled for the next observing season. We also plan to make the system available for regular observation campaigns beginning with the 2013 observing season.

Acknowledgements This project is in part funded by the Leibniz-Gemeinschaft within the "Pakt für Forschung und Innovation”. A. Fischer, K. Gerber, T. Sonner, and M. Weissschädel provided invaluable technical support for the setup at the VTT.

\section{References}

Balthasar, H.: 1984, Solar Phys. 93, 219. doi:10.1007/BF02270836.

Beckers, J.M., Nelson, G.D.: 1978, Solar Phys. 58, 243. doi:10.1007/BF00157270.

Benedick, A.J., Chang, G., Birge, J.R., Chen, L.-J., Glenday, A.G., Li, C.-H., Phillips, D.F., Szentgyorgyi, A., Korzennik, S., Furesz, G., Walsworth, R.L., Kärtner, F.X.: 2010, Optics Express 181, 19175. doi:10.1364/OE.18.019175.

Black, E.D.: 2001, American Journal of Physics 69, 79. doi:10.1119/1.1286663.

Braje, D.A., Kirchner, M.S., Osterman, S., Fortier, T., Diddams, S.A.: 2008, The European Physical Journal D - Atomic, Molecular, Optical and Plasma Physics 48, 57. 10.1140/epjd/e2008-00099-9. http://dx.doi.org/10.1140/epjd/e2008-00099-9.

Brault, J.W.: 1987, Mikrochimica Acta 3, 215.

de la Cruz Rodríguez, J., Kiselman, D., Carlsson, M.: 2011, Astron. Astrophys. 528, A113. doi:10.1051/0004-6361/201015664.

Dikpati, M., Gilman, P.A.: 2007, New Journal of Physics 9, 297. doi:10.1088/1367-2630/9/8/297. 
Holzwarth, R., Udem, T., Hänsch, T.W., Knight, J.C., Wadsworth, W.J., Russell, P.S.J.: 2000, PRL 85, 2264.

Kentischer, T.J., Schroeter, E.H.: 1991, Astron. Astrophys. 245, 279.

Koch, A., Woehl, H.: 1984, Astron. Astrophys. 134, 134.

Lagrange, A.-M., Desort, M., Meunier, N.: 2010, Astron. Astrophys. 512, A38. doi:10.1051/00046361/200913071

Li, C.-H., Benedick, A.J., Fendel, P., Glenday, A.G., Kärtner, F.X., Phillips, D.F., Sasselov, D., Szentgyorgyi, A., Walsworth, R.L.: 2008, Nature 452, 610. doi:10.1038/nature06854

Lovis, C., Pepe, F.: 2007, Astron. Astrophys. 468, 1115. doi:10.1051/0004-6361:20077249

Lovis, C., Mayor, M., Pepe, F., Queloz, D., Udry, S.: 2008, In: D. Fischer, F. A. Rasio, S. E. Thorsett, \& A. Wolszczan (ed.) Extreme Solar Systems, Astronomical Society of the Pacific Conference Series 398, 455.

Makarov, V.V.: 2010, Astrophys. J. 715, 500. doi $10.1088 / 0004-637 X / 715 / 1 / 500$.

Murphy, M.T., Udem, T., Holzwarth, R., Sizmann, A., Pasquini, L., Araujo-Hauck, C., Dekker, H., D'Odorico, S., Fischer, M., Hänsch, T.W., Manescau, A.: 2007, Mon. Not. Roy. Astron. Soc 380, 839.

Stark, S.P., Steinmetz, T., Probst, R.A., Hundertmark, H., Wilken, T., Hänsch, T.W., Udem, T., Russell, P.S.J., Holzwarth, R.: 2011, Opt. Express 19(17), 15690. doi:10.1364/OE.19.015690 http://www.opticsexpress.org/abstract.cfm?URI=0e-19-17-15690.

Steinmetz, T., Wilken, T., Araujo-Hauck, C., Holzwarth, R., Hänsch, T.W., Pasquini, L., Manescau, A., D’Odorico, S., Murphy, M.T., Kentischer, T., Schmidt, W., Udem, T.: 2008, Science 321.

Steinmetz, T., Wilken, T., Araujo-Hauck, C., Holzwarth, R., Hänsch, T.W., Udem, T.: 2009, Applied Physics B: Lasers and Optics 96, 251. doi:10.1007/s00340-009-3374-6

Wilken, T.: 2010, Calibrating astronomical spectrographs with frequency combs. PhD thesis, LMU München.

Wilken, T., Lovis, C., Manescau, A., Steinmetz, T., Pasquini, L., Lo Curto, G., Hänsch, T.W., Holzwarth, R., Udem, T.: 2010, Mon. Not. Roy. Astron. Soc 405, L16. doi:10.1111/j.1745-3933.2010.00850.x. 